\title{
Bancos de cholga Aulacomya atra atra (Molina) (Bivalvia: Mytilidae) del golfo San José (Chubut, Argentina): Diversidad y relaciones con facies afines
}

\author{
Ribbed mussel Aulacomya atra atra (Molina) (Bivalvia: Mytilidae) beds of the San José Gulf \\ (Chubut, Argentina): Diversity and relationships with related facies
}

\author{
Héctor E. Zaixso
}

\author{
Consejo Nacional de Investigaciones Científicas y Técnicas. Universidad Nacional de la Patagonia \\ S. J. Bosco. Km 4 (9000) Comodoro Rivadavia, Chubut, Argentina \\ hzaixso@uolsinectis.com.ar
}

\begin{abstract}
Resumen.- Se estudió la diversidad y relaciones entre diferentes poblamientos de la biocenosis de raspadoressuspensívoros del golfo San José (Chubut, Argentina: 42²0'S; $\left.64^{\circ} 20^{\prime} \mathrm{W}\right)$, con énfasis en los bancos de cholga Aulacomya atra atra. Las relaciones entre poblamientos se estudiaron a través de un análisis de correspondencias sin tendencias y las relaciones entre poblamientos y factores ambientales a través de un análisis factorial de correspondencias. Para el estudio de la diversidad se utilizaron los índices de diversidad de Hill $\left(\mathrm{N}_{0}, \mathrm{~N}_{1} \mathrm{y} \mathrm{N}_{2}\right)$, así como el índice de uniformidad derivado $\left(\mathrm{E}=\mathrm{N}_{2} / \mathrm{N}_{1}\right)$. Se analizaron, dentro del contexto de estos poblamientos, las relaciones entre la diversidad observada con la resistencia a los disturbios, la presencia de depredadores y con la sucesión y aumento de la heterogeneidad espacial. Se concluyó que: (1) Los bancos de cholga sobre sustratos duros, en razón de hallarse en sitios protegidos del oleaje producido por vientos locales y con una matriz física fijada firmemente a la roca mantienen una alta riqueza específica y una alta uniformidad. (2) Los poblamientos de fondos blandos de mejillón Mytilus edulis platensis en condiciones de estabilidad, evolucionan hacia bancos mixtos con cholgas lo que implica aumento en la complejidad del sustrato, en la heterogeneidad espacial del banco y en la riqueza específica. Estos bancos mixtos al quedar sujetos a disturbios físicos revertirían el proceso de sucesión. (3) Los bancos de vieira tehuelche Aequipecten tehuelchus son propios de ambientes permanentemente disturbados por movimientos del agua y poseen características de baja uniformidad y baja riqueza específica. Con disturbios intensos e infrecuentes estos bancos son eliminados del sitio que ocupaban, ocasionándose arribazones de las especies desprendidas. (4) Los bancos mixtos de cholga-vieira tehuelche son facies alternativas a los bancos de vieira y tienen lugar en sitios protegidos.
\end{abstract}

Palabras clave: Poblamientos, complejidad estructural, disturbios, estabilidad, sucesión

\begin{abstract}
The relationships between different assemblages of the grazer-suspensivore biocoenosis and their diversities were studied at the San José Gulf (Chubut Province, Argentina: $42^{\circ} 20^{\prime} S$; $64^{\circ} 20^{\prime} \mathrm{W}$ ), with emphasis on the facies of the ribbed mussel Aulacomya atra atra. The ordination analysis of the assemblages species composition was performed using detrended correspondence analysis and the relationships of the assemblages with the environmental factors were analysed using factorial correspondence analysis. The Hill diversity indices $\left(\mathrm{N}_{0}, \mathrm{~N}_{1}, \mathrm{~N}_{2}\right)$, and the evenness derived index $\left(E=N_{2} / N_{1}\right)$ were calculated. It was concluded that: (1) The ribbed mussel beds on hard sustrate have high species richness and high equitability, because the rocks provides firm surfaces for byssal attachment and these beds are usually found in protected localities. (2) The soft bottom assemblages with the mussel Mytilus edulis platensis, under conditions of environmental stability, evolve towards mixed beds with ribbed mussel; this process implies increased sustrate complexity, increased spatial heterogeneity and higher species richness. This successional process can be reverted if the mixed bed are affected by strong physical disturbance which can dislodge the assemblages. (3) Beds of the scallop Aequipecten tehuelchus are found at sites permanently disturbed by waves generated by local winds, they have low equitability and low species richness. When the environmental disturbance is very intense the bed is dislodged and big quantities of individuals are beach stranded. (4) The mixed beds of ribbed mussel and Aequipecten tehuelchus are facies which are alternative to Aequipecten beds and they are found in protected sites.
\end{abstract}

Key words: assemblages, structural complexity, disturbances, stability, succession 


\section{Introducción}

De acuerdo a Pérès \& Picard (1964) se define a una facies como un aspecto de una biocenosis donde predominan, debido a condiciones locales específicas, una especie o un bajo número de ellas, las cuales pueden o no ser indicadoras de la biocenosis. Esta definición, cuando las especies dominantes o codominantes, tienen importancia pesquera, coincide con el concepto de "banco", término que es utilizado en la Argentina, para describir las poblaciones de mariscos y peces comerciales y las de sus especies acompañantes.

A diferencia de la de facies o bancos, la definición de poblamiento, no implica necesariamente la dominancia de una o más especies, correspondiendo a un subconjunto funcional de poblaciones de diferentes especies que: (1) es parte de una misma biocenosis; (2) tiene un nivel de organización intermedio entre las poblaciones y la biocenosis; (3) puede ser objeto de estudios analíticos simplificados y (4) sus componentes deben ser establecidos para cada caso en particular (Barbault 1981).

En un estudio sobre las asociaciones del macrozoobentos submareal del golfo San José, Zaixso et al. (1998) describen una biocenosis de raspadoressuspensívoros, la que caracterizan por la presencia de: Aulacomya atra atra, los poliquetos Eunice argentinensis y Harmothoe magellanica, los bivalvos Pododesmus rudis, Aequipecten tehuelchus y Zygochlamys patagonica, el gasterópodo Fissurella radiosa tixierae y los equinodermos Arbacia dufresnei y Ophiactis asperula. Esta biocenosis, que se define en términos cualitativos, abarca, entre otras facies, a las que comúnmente se denominan "bancos de cholga" y "bancos de vieira", los que son objeto de pesca comercial en el golfo; es decir que dichos bancos representan sólo algunas de las fisonomías cuantitativas que puede adoptar la biocenosis (Zaixso et al. 1998). La composición cualitativa y cuantitativa de los bancos de vieira es estudiada por Ciocco (1988), en tanto que los bancos de mejillón, comprendidos dentro de la misma biocenosis, son descriptos por Bala (1989).

Los bancos de la cholga Aulacomya atra atra (Molina 1782), de la costa argentina, ubicados entre el golfo San Matías y Tierra del Fuego, constituyen una fisonomía corriente en los fondos rocosos o de granulometría muy gruesa del piso infralitoral (Olivier et al. 1966, Otaegui \& Zaixso 1974, Urquiola de Carli \& Aramayo 1979, Barrionuevo et al. 1976, Zaixso \& Pastor 1978, Escofet et al. 1978, Zaixso et al. 1979, Carriquiriborde et al. 1983, Zaixso 1996b, Zaixso et al.
1998, Zaixso 1999). Se encuentran también bancos de cholga a lo largo de la costa de Chile y sur de Perú, donde la mayor parte de los bancos estudiados se ubican entre los 0 y los $25 \mathrm{~m}$ de profundidad, preferentemente sobre fondos rocosos (Cancino \& Becerra 1978). Eventualmente, bajo condiciones ambientales apropiadas, los bancos de Aulacomya atra atra pueden ser encontrados también en el piso circalitoral (Zaixso 1999).

Los bancos de cholga son el asiento de una comunidad altamente diversa, donde la cholga actúa como sustrato de numerosas especies de algas y animales y es alimento de gasterópodos, asteroideos y aves marinas y hospedador de varias especies de organismos parásitos, comensales y perforantes (Zaixso 2003) ${ }^{1}$. Entre los trabajos específicos dedicados a la ecología de Aulacomya atra atra en la costa argentina, se pueden indicar varios referidos a la "cholguera" de punta Loma en golfo Nuevo (Urquiola de De Carli \& Aramayo 1970, Penchaszadeh et al. 1974, Barrionuevo et al. 1976) y uno más reciente, donde se estudia la distribución submareal de este mitílido en el golfo San José y algunos de los factores que la regulan (Zaixso 1999).

A pesar de la importancia ecológica que tienen los bancos de cholga y otras facies afines, las causas de su diversidad no han sido analizadas hasta el presente. Este trabajo tiene por objetivos: (1) el análisis de la composición, con énfasis en los bancos de cholga, de los diferentes poblamientos submareales de la biocenosis de raspadores-suspensívoros del golfo San José (provincia del Chubut, Argentina), tanto de aquellos con dominancia de algunas especies, correspondientes a facies o bancos, como aquellos donde no se observan dominancias; (2) la comparación de la diversidad de dichos poblamientos y (3) la discusión de los efectos de los disturbios, de la presencia de depredadores, de la sucesión ecológica y de la heterogeneidad espacial, sobre su diversidad.

\section{Materiales y métodos}

El presente estudio fue llevado a cabo en el golfo San José, Argentina (42 $\left.20^{\prime} \mathrm{S} ; 6^{\circ} 20^{\prime} \mathrm{W}\right)$. El golfo es de forma aproximadamente rectangular y mide unos 42 $\mathrm{Km}$ de este a oeste por $19 \mathrm{Km}$ de norte a sur; su profundidad media es de unos $30 \mathrm{~m}$, con profundidades máximas del orden de los $85 \mathrm{~m}$. La temperatura

\footnotetext{
${ }^{1}$ Zaixso HE. 2003. Ecología de la cholga, Aulacomya atra atra. XXIII Congreso de Ciencias del Mar. Punta Arenas, Chile, p.51, resumen.
} 
superficial del agua varía entre $9,8^{\circ} \mathrm{C}$ en invierno y $16,5^{\circ} \mathrm{C}$ en verano; la salinidad presenta escasas variaciones siendo en promedio de 33,82 psu. Los vientos dominantes provienen principalmente del oeste y sudoeste, alcanzando velocidades de hasta $7 \mathrm{~m}$ por segundo, en consecuencia, las costas norte y este son las más expuestas al oleaje producido por vientos fuertes. Las asociaciones del macrozoobentos y de macroalgas, definidas en ambos casos cualitativamente, son descriptas en Zaixso et al. (1998) y Boraso et al. (1999) respectivamente. Los aspectos referidos a tipos de fondo, temperaturas del agua, corrientes y otros parámetros físico-químicos de las áreas muestreadas son detallados en Charpy \& Charpy (1977), Ciocco (1985, 1988), Bala (1989), Zaixso (1993, 1996a, 1996b, 1999) y Zaixso et al. (1998).

Se adoptó como criterio de inclusión en el universo a examinar, que las unidades muestrales (u.m.) provinieran del área ocupada por la biocenosis submareal de raspadores-suspensívoros del golfo San José, definida en Zaixso et al. (1998). Las u.m. fueron tomadas dentro de una amplia variedad de ambientes en los que se pueden diferenciar, de acuerdo a la dominancia en abundancia de las especies más conspicuas, a lo que en el presente trabajo denominaremos facies o bancos. En la zona de estudio se han podido diferenciar los siguientes tipos de facies: (1) bancos de cholga Aulacomya atra atra, (2) bancos de mejillón Mytilus edulis platensis, (3) bancos mixtos de mejillón y cholga, (4) bancos de vieira tehuelche Aequipecten tehuelchus, (5) bancos mixtos de vieira y cholga, (6) bancos de vieira patagónica Zygochlamys patagonica y (7) bancos de almeja rayada Ameghinomya antiqua (=Venus antiqua). En adición, fueron incluidas u.m. en las que no pudo observarse la dominancia de alguna especie en particular y que denominamos (8) poblamientos sin dominancias.

Las u.m. utilizadas $(n=130)$, de $1 / 4 \mathrm{~m}^{2}$ de superficie, fueron obtenidas durante diferentes muestreos y con las técnicas apropiadas para cada caso. Las u.m. de bancos de cholgas de aguas someras (hasta unos $10 \mathrm{~m}$ de profundidad en bajamar) (punta Gales, punta Mejillón), de mejillones (El Bote, El Riacho San José y punta Logaritmo), de vieiras (fondeadero San Román, punta Tehuelche) y de áreas de transición entre éstos o hacia otras biocenosis del golfo (punta Juan de La Piedra, Juan de La Piedra-Gales), fueron obtenidas mediante buceo autónomo durante los meses de mayo a septiembre de 1985. Las u.m. de bancos profundos (más de 15-20 m), se obtuvieron con draga durante las campañas San José I (agosto a septiembre de 1984) y San José II (diciembre de 1984).
La elaboración de las u.m. consistió en el lavado de éstas sobre un tamiz de $1 \mathrm{~mm}$ de abertura y en la separación, identificación y conteo de los ejemplares de la macrofauna hallados. Los anfípodos no fueron incluidos en el análisis.

Las u.m. fueron analizadas en primer lugar, mediante un análisis de correspondencias sin tendencias (DCA), utilizando para ello el programa CANOCO 4.0 (ter Braak \& Smilauer 1998) con los siguientes parámetros: datos sin transformar, eliminación de tendencias mediante la técnica de segmentos, preservación de la distancia de $\chi^{2}$ entre las u.m. y "biplot scaling”. Para evitar la influencia excesiva de las especies raras sobre la ordenación se utilizó la opción “down-weigthing” de las mismas.

La interpretación del diagrama de ordenación se efectuó de acuerdo a la regla de distancia. Esta regla es una extensión del principio del centroide y expresa que dos u.m. son más semejantes, cuanto más cercanas se hallan entre sí; que una u.m. que se halla cercana a una especie es más probable que contenga a esa especie que una u.m. que está lejos de ella y que el rango de las abundancias de varias especies en una u.m. se puede inferir de la distancia de las especies a la u.m. (ter Braak \& Smilauer 1998). Al utilizar la opción de “scaling” con énfasis en la distancia entre u.m., los puntos de las especies se disponen rodeando a los de las u.m. y las especies que se encuentran alrededor de una u.m. en particular, participan en su composición en una relación aproximadamente proporcional a su distancia a la misma.

La relación de los poblamientos con la profundidad, exposición a vientos y olas y tipo de sustrato fue estudiada a través de un análisis factorial de correspondencias (CA) sobre una tabla de contingencias (Legendre \& Legendre 1998). En esta situación, el CA compara las diferentes descripciones de un primer conjunto de descriptores (tipos de poblamientos) con las de un segundo conjunto de descriptores (variables ambientales en clases). Las celdas de la tabla contienen el número de objetos (u.m.) correspondientes a cada combinación de descriptores y una u.m. puede contribuir a varias de las frecuencias de la tabla (Legendre \& Legendre 1998). Las profundidades de las u.m. referidas al nivel del plano de reducción local fueron divididas en las siguientes clases: pro1 (0 a 4,9 m), pro2 (5 a 14,9 m), pro3 (15 a 29,9 m) y pro4 (30 o más metros). La exposición al oleaje producido por vientos locales se dividió en tres clases: baja (costa oeste y mitad occidental de la costa sur del golfo), media (mitad oriental de la costa sur y parte de la zona central) y alta (costas norte y este del golfo y parte de la 
zona central). El tipo de sustrato fue dividido en cuatro clases: Fondos de toba (sustrato duro), fondos S1-S2 (sustratos con predominio de las fracciones de gránulos $\mathrm{y}$ arenas muy gruesas), fondos S3 (sustratos con predominio de arenas finas) y fondos S4-S5, (sustratos con dominancia de las fracciones de arenas muy finas a limos y arcillas); una descripción más completa de estos tipos de fondo y de la metodología usada en su diferenciación se encuentra en Zaixso (1996b).

Para cada una de las u.m. se calcularon los tres primeros órdenes de los índices de diversidad de Hill (1973), $N_{0}, N_{1}$ y $N_{2}$, así como el índice de uniformidad derivado $E=N_{2} / N_{1}$. En conjunto, estos índices han demostrado ser más fácilmente interpretables que otros índices en uso (Ludwig \& Reynolds 1988).

Los índices de Hill constituyen una familia de índices que bajo su forma general son:

$$
\begin{aligned}
& \mathrm{N}_{\mathrm{a}}=\left(\mathrm{p}_{1}{ }^{\mathrm{a}}+\mathrm{p}_{2}{ }^{\mathrm{a}}+\mathrm{p}_{3}{ }^{\mathrm{a}}+\ldots+\mathrm{p}_{\mathrm{n}}{ }^{\mathrm{a}}\right)^{1 / 1-\mathrm{a}} \\
& \text { donde: }
\end{aligned}
$$

a es el orden del índice de diversidad

$\mathrm{p}_{\mathrm{n}}$ es la proporción de la abundancia de la especie n

De lo anterior se desprende que cuando $\mathrm{a}=0$ entonces $\mathrm{N}_{0}$ es igual al número total de especies en la u.m. De manera semejante se obtiene que $\mathrm{N}_{1}$ es igual al índice de Shannon exponencial $\left(\mathrm{N}_{1}=\mathrm{e}^{\mathrm{H}^{\prime}}\right)$ y que $\mathrm{N}_{2}$ es igual a la inversa del índice de Simpson $\left(\mathrm{N}_{2}=1 / \mathrm{D}\right)$ (Ludwig \& Reynolds 1988, Magurran 1988). Los índices de Hill, cuyas unidades se hallan expresadas en número de especies, miden el grado en que las proporciones de abundancia se hallan distribuidas entre las especies y a medida que su orden aumenta, es menor el peso que el índice pone en las especies raras (Alatalo \& Alatalo 1977). De esta manera $\mathrm{N}_{0}$ toma en cuenta a todas las especies, $\mathrm{N}_{1}$ mide el número efectivo de las especies abundantes y $\mathrm{N}_{2}$ mide el número efectivo de las especies muy abundantes (Ludwig \& Reynolds 1988). El índice de uniformidad $\mathrm{E}=\mathrm{N}_{2} / \mathrm{N}_{1}$ fue propuesto por Hill (1973) y representa el cociente entre el número de especies muy abundantes y las abundantes; a medida que una especie tiende a dominar en un poblamiento, tanto $\mathrm{N}_{2}$ como $\mathrm{N}_{1}$ tienden hacia uno y en consecuencia también E converge hacia este valor.

Con los valores de diversidad obtenidos se llevó a cabo un análisis de la varianza (ANOVA) entre poblamientos, exceptuando de este análisis a aquellos con un escaso número de u.m. (facies de Zygochlamys patagonica y de Ameghinomya antiqua). Previo a estos análisis, se llevó a cabo una prueba log-anova de homogeneidad de varianzas (Sokal \& Rholf 1997), debiéndose en una de las circunstancias $\left(\mathrm{N}_{0}\right)$, practicarse la transformación de los datos por $\mathrm{X}^{\prime}=\mathrm{X}^{0,5}$. Los análisis con resultado significativo fueron seguidos de una prueba GT2 de comparación múltiple de medias (Sokal \& Rholf 1997).

La complejidad estructural (física) de los poblamientos fue descripta cualitativamente a partir de observaciones directas restringidas a aquellos ubicados a poca profundidad.

Las observaciones sobre la estructura y composición de bancos profundos de Zygochlamys patagonica, en base a las cuales se describe brevemente a los mismos, fueron realizadas sobre muestras provenientes de dragados no disturbados y de rastreos cualitativos, estos últimos no incluidos en los análisis cuantitativos precedentes, efectuados durante las campañas San José I y San José II.

\section{Resultados}

\section{Composición en especies y grupos tróficos}

En la Tabla 1 se describe en forma sumaria la composición en especies de las facies y poblamientos componentes de la biocenosis de raspadores y suspensívoros, indicándose las estimaciones de algunos parámetros de tendencia central y dispersión para las especies principales (presentes en al menos 50\% de las u.m.) de cada uno de ellos La composición específica cualitativa fue notablemente constante en los diferentes poblamientos analizados, quedando caracterizado cada uno de ellos por la mayor abundancia de la especie que le da el nombre; excepciones a esto fueron los bancos de vieira patagónica, donde la especie más abundante fue el cirripedio Austromegabalanus pssittacus, y los poblamientos sin dominancias. Aulacomya atra atra se encontró entre las principales especies de los bancos de cholga, vieira tehuelche, mixtos de cholga-mejillón y cholga-vieira tehuelche $\mathrm{y}$ en los poblamientos sin dominancias.

La abundancia porcentual de los distintos grupos tróficos presentes se describe en la Tabla 2. En líneas generales se pudo observar que las especies suspensívoras de epifauna fueron abundantes en todos los poblamientos, incluyendo a bivalvos (por ej. Aulacomya atra atra, Mytilus edulis platensis, Aequipecten tehuelchus, Pododesmus rudis $\mathrm{y}$ Zygochlamys patagonica), gasterópodos (principalmente Crepipatella dilatata) y otros 
suspensívoros (por ej. Paramolgula gregaria, Aerothyris venosa, Chaetopterus aff. variopedatus). Las especies suspensívoras de infauna (por ej. Ameghinomya antiqua) fueron más escasas. Los raspadores, como los poliplacóforos (Leptochiton medinae, Chaetopleura isabellei), gasterópodos (Lepeta coppingeri, Tegula patagonica y Fissurella radiosa tixierae) y los macroherbívoros (los erizos regulares Arbacia dufresnei y Pseudechinus magellanicus), se hallaron bien representados en todos los poblamientos, excepto en los bancos de mejillón, donde sólo se encontró a estos últimos. Hubo una baja representación de especies consideradas como depredadores típicos de bivalvos; el caracol murícido Trophon geversianus sólo se halló presente en los bancos de cholga o en los mixtos de cholga-mejillón; el asteroideo Anasterias minuta se encontró en los bancos de cholga, de cholga- mejillón y de cholga-vieira y los asteroideos, Cosmasterias lurida y Allostichaster capensis sólo se encontraron en bancos de cholga. Los poliquetos carnívoros (Eunice argentinensis y Harmothoe magellanica) y nemertinos en cambio, se hallaron bien representados en casi todos los poblamientos; estas especies no son consideradas depredadores de bivalvos. La mayor cantidad de especies depositívoras (los bivalvos Macoma uruguayensis y Malletia cumingi y poliquetos Terebellidae y Cirratulidae) se encontró en los bancos de almeja, vieira patagónica y cholga. Las especies omnívoras, en su mayoría crustáceos decápodos (por ej. Leucippa pentágona y Peltarium spinosulum), se encontraron representadas en todos los poblamientos, con un máximo de especies en los bancos de cholga.

Tabla 1

Composición en especies de los poblamientos. Especies presentes en al menos un $\mathbf{5 0 \%}$ de las unidades muestrales (u.m.) de los bancos o poblamientos, ordenadas por el rango de las medias del número de individuos por $1 / 4 \mathbf{m}^{2}$. N, número de u.m.

Assemblages species composition. Species present in each bed or assemblage at least in $50 \%$ of the sampling units (s.u.). The species are ordered by the rank of the mean of individual number $\left(1 / 4 \mathrm{~m}^{2}\right)$. N, number of s.u.

\begin{tabular}{lcccc}
\hline Bancos de cholga $(\mathrm{N}=39)$ & Media & Error estándar & Mediana & Rango \\
Especie & 133,32 & 30,36 & 59,0 & $4-1.040$ \\
Aulacomya atra atra & 10,00 & 4,23 & 2,0 & $0-141$ \\
Ophiactis asperula & 8,66 & 2,29 & 2,5 & $0-72$ \\
Tegula patagonica & 4,08 & 0,59 & 3,5 & $0-15$ \\
Eunice argentinensis & 3,79 & 1,27 & 1,0 & $0-37$ \\
Nemertina & 2,87 & 0,55 & 1,5 & $0-13$ \\
Paramolgula gregaria & 2,79 & 0,73 & 1,0 & $0-20$ \\
Platynereis sp. & 2,68 & 0,44 & 2,0 & $0-11$ \\
Arbacia dufresnei & 2,03 & 0,42 & 1,0 & $0-10$ \\
Cirratulidae & 1,68 & 0,29 & 1,0 & $0-8$ \\
Pododesmus rudis & 1,63 & 0,30 & 1,0 & $0-7$ \\
Harmothoe magellanica & 1,40 & 0,39 & 1,0 & $0-7$ \\
Fissurella radiosa tixierae & 0,97 & 0,19 & 1,0 & $0-4$ \\
Allostichaster capensis & & & \\
\hline & & & \\
\hline Bancos de mejillón $(\mathrm{N}=11)$ & Media & Error estándar & Mediana & Rango \\
Especie & 12,09 & 0,76 & 10,0 & $5-24$ \\
Mytilus edulis platensis & 10,18 & 1,77 & 9,0 & $1-21$ \\
Crepidula protea & 1,55 & 0,39 & 2,0 & $0-4$ \\
Platynereis sp. & 1,09 & 0,32 & 1,0 & $0-3$ \\
Paramolgula gregaria & 0,55 & 0,16 & 1,0 & $0-1$ \\
Harmothoe magellanica & & & \\
\hline
\end{tabular}


continuación Tabla 1

Bancos mixtos de cholga-mejillón ( $\mathrm{N}=15)$
Especie

Aulacomya atra atra

Mytilus edulis platensis

Platynereis sp.

Crepipatella dilatata

Nemertina

Cirratulidae

Terebellidae

Lumbrineridae

Harmothoe magellanica

Chaetopleura isabellei

$\begin{array}{rc}\text { Media } & \text { Error estándar } \\ 53,07 & 9,54 \\ 30,53 & 5,60 \\ 13,00 & 2,44 \\ 9,87 & 1,36 \\ 6,00 & 2,71 \\ 4,80 & 0,90 \\ 3,33 & 1,04 \\ 3,27 & 0,52 \\ 1,73 & 0,38 \\ 1,07 & 0,25\end{array}$

Media

1,07

$\begin{array}{cc}\text { Mediana } & \text { Rango } \\ 55,0 & 6-104 \\ 28,0 & 3-75 \\ 9,0 & 3-30 \\ 9,0 & 2-25 \\ 1,0 & 0-33 \\ 5,0 & 0-11 \\ 2,0 & 0-11 \\ 3,0 & 0-6 \\ 2,0 & 0-5 \\ 1,0 & 0-3\end{array}$

Bancos de vieira tehuelche $(\mathrm{N}=22)$

\section{Especie}

Aequipecten tehuelchus

Terebellidae

Tegula patagonica

Platynereis sp.

Paramolgula gregaria

Eunice argentinensis

Chaetopleura isabellei

Aulacomya atra atra

Harmothoe magellanica

Fissurella radiosa tixierae

Ophioplocus januari

Media

12,71

6,14

6,10

5,81

4,81

4,43

3,38

2,43

1,43

1,26

1,14

\section{Error estándar}

1,09

1,70

1,54

1,47

1,12

1,08

0,88

0,83

0,53

0,27

0,29

\section{Mediana}

12,0

3,0

2,0

4,0

4,0

4,0

2,0

1,0

1,0

1,0
Rango

$5-22$

$0-27$

$0-23$

$0-29$

0 - 19

0 - 22

0 - 19

$0-15$

$0-11$

$0-4$

$0-5$

Bancos mixtos de cholga-vieira tehuelche $(\mathrm{N}=8)$

\section{Especie}

Aulacomya atra atra

Terebellidae

Aequipecten tehuelchus

Crepidula aculeata

Fissurella radiosa tixierae

Platynereis sp.

Cirratulidae

Paramolgula gregaria

Eunice argentinensis

Chaetopleura isabellei

Lepeta coppingeri

Arbacia dufresnei

Harmothoe magellanica

Pseudechinus magellanicus

Tegula patagonica

Nemertina
Media

33,25

12,38

7,88

5,50

4,13

3,88

3,00

2,88

2,88

2,75

2,50

1,75

1,25

0,88

0,63

0,50

\section{Error estándar}

5,21

2,85

0,40

1,18

1,08

0,81

0,66

1,06

1,06

0,96

0,80

0,41

0,37

0,40

0,26

0,19
Mediana

37,5

10,5

8,0

5,0

3,0

3,0

3,0

2,0

1,5

2,0

2,0

1,5

1,0

0,5

0,5

0,5

\section{Rango}

6 - 49

$5-30$

$6-9$

$2-11$

$0-9$

2- 9

$0-6$

0 - 10

0 - 9

$0-9$

$0-6$

$0-3$

$0-3$

$0-3$

$0-2$

$0-1$

Bancos de almeja rayada $(\mathrm{N}=7)$

\section{Especie}

Ameghinomya antiqua

Eunice argentinensis

Arbacia dufresnei

Harmothoe magellanica

\begin{tabular}{rc} 
Media & Error estándar \\
6,14 & 2,44 \\
1,71 & 0,68 \\
1,71 & 0,68 \\
1,57 & 0,65 \\
\hline
\end{tabular}

Mediana

Rango

3,0

2 - 20

1,0

$0-5$

1,0

$0-5$

1,0

$0-4$ 
continuación Tabla 1

\begin{tabular}{|c|c|c|c|c|}
\hline \multicolumn{5}{|c|}{ Bancos de vieira patagónica $(\mathrm{N}=4)$} \\
\hline Especie & Media & Error estándar & Mediana & Rango \\
\hline Austromegabalanus pssittacus & 34,25 & 22,19 & 20,5 & $0-96$ \\
\hline Zygochlamys patagonica & 6,50 & 1,56 & 5,5 & $4-11$ \\
\hline Eunice argentinensis & 3,75 & 2,06 & 3,0 & $0-9$ \\
\hline Ophioplocus januari & 3,50 & 2,36 & 2,0 & $0-10$ \\
\hline Corbula lyon & 1,50 & 0,96 & 1,0 & $0-4$ \\
\hline Arbacia dufresnei & 0,75 & 0,48 & 0,5 & $0-2$ \\
\hline Ameghinomya antiqua & 0,75 & 0,48 & 0,5 & $0-2$ \\
\hline Chaetopterus aff. variopedatus & 0,75 & 0,48 & 0,5 & $0-2$ \\
\hline Macoma uruguayensis & 0,75 & 0,48 & 0,5 & $0-2$ \\
\hline Harmothoe magellanica & 0,50 & 0,29 & 0,5 & $0-1$ \\
\hline \multicolumn{5}{|c|}{ Poblamientos sin dominancias $(\mathrm{N}=24)$} \\
\hline Especie & Media & Error estándar & Mediana & Rango \\
\hline Pododesmus rudis & 3,21 & 0,59 & 2,5 & $0-10$ \\
\hline Eunice argentinensis & 2,54 & 0,60 & 1,0 & $0-9$ \\
\hline Aulacomya atra atra & 2,33 & 0,86 & 1,0 & $0-15$ \\
\hline Paramolgula gregaria & 2,00 & 0,56 & 1,0 & $0-9$ \\
\hline Chaetopleura isabellei & 1,13 & 0,27 & 1,0 & $0-5$ \\
\hline Harmothoe magellanica & 1,04 & 0,21 & 1,0 & $0-3$ \\
\hline Aequipecten tehuelchus & 0,92 & 0,24 & 1,0 & $0-4$ \\
\hline
\end{tabular}

Tabla 2

Grupos tróficos en cada tipo de banco o poblamiento. Solo se consideran aquellas especies donde los hábitos alimentarios han sido confirmados. N, número de especies; \%, porcentaje del número de individuos; CH, bancos de cholga (Aulacomya atra atra); M, bancos de mejillón (Mytilus edulis platensis); CH-M, bancos de cholga y mejillón; V, bancos de vieira tehuelche (Aequipecten tehuelchus); CH-V, bancos de cholga y vieira tehuelche; A, bancos de almeja (Ameghinomya antiqua); VP, bancos de vieira patagónica (Zygochlamys patagonica); SD, poblamientos sin dominancias

Trophic groups present in the assemblages. Only the species whose feeding behaviour is known are included. N, species number; \%, percentage of individuals; CH, ribbed mussel beds (Aulacomya atra atra); M, blue mussel beds (Mytilus edulis platensis); CH-M, ribbed mussel - blue mussel beds; $\mathrm{V}$, tehuelche scallop beds (Aequipecten tehuelchus); $\mathrm{CH}-\mathrm{V}$, ribbed mussel - tehuelche scallop beds;

A, clam beds (Ameghinomya antiqua); VP, patagonic scallop beds (Zygochlamys patagonica); SD, no dominance assemblages

\begin{tabular}{|c|c|c|c|c|c|c|c|c|c|}
\hline \multirow{3}{*}{$\begin{array}{l}\text { Bivalvos suspensívoros } \\
\text { epifauna }\end{array}$} & \multicolumn{2}{|c|}{$\mathbf{C H}$} & \multirow{2}{*}{$\begin{array}{c}\mathbf{M} \\
1\end{array}$} & \multirow{2}{*}{$\begin{array}{c}\text { Ch-M } \\
3\end{array}$} & \multirow{2}{*}{$\begin{array}{l}\mathbf{V} \\
3\end{array}$} & \multirow{2}{*}{$\begin{array}{c}\text { Ch-V } \\
4\end{array}$} & \multirow{2}{*}{$\begin{array}{c}\mathbf{A} \\
3\end{array}$} & \multirow{2}{*}{$\begin{array}{c}\mathbf{V P} \\
3\end{array}$} & \multirow{2}{*}{$\begin{array}{c}\text { SD } \\
5\end{array}$} \\
\hline & $\mathrm{N}$ & 5 & & & & & & & \\
\hline & $\%$ & 71,57 & 46,02 & 72,94 & 28,05 & 48,30 & 11,61 & 13,88 & 17,67 \\
\hline Bivalvos suspensívoros & $\mathrm{N}$ & 1 & ----- & ----- & ----- & 2 & 3 & 2 & 1 \\
\hline infauna & $\%$ & 0,11 & & & & 0,30 & 17,42 & 3,67 & 0,46 \\
\hline Gasterópodos & $\mathrm{N}$ & 3 & 3 & 2 & 1 & 1 & 2 & ----- & 3 \\
\hline suspensívoros & $\%$ & 0,82 & 40,85 & 9,26 & 1,51 & 6,40 & 1,03 & & 1,10 \\
\hline \multirow[t]{2}{*}{ Otros suspensívoros } & $\mathrm{N}$ & 5 & 1 & 1 & 4 & 3 & 5 & 5 & 6 \\
\hline & $\%$ & 8,28 & 4,15 & 0,46 & 24,37 & 3,65 & 16,72 & 65,31 & 37,32 \\
\hline \multirow[t]{2}{*}{ Gasterópodos raspadores } & $\mathrm{N}$ & 3 & ----- & 3 & 3 & 3 & 3 & 1 & 3 \\
\hline & $\%$ & 5,79 & & 1,74 & 13,56 & 5,82 & 18,80 & 0,41 & 22,21 \\
\hline \multirow[t]{2}{*}{ Poliplacóforos } & $\mathrm{N}$ & 3 & ----- & 1 & 2 & 2 & 2 & 1 & 3 \\
\hline & $\%$ & 2,54 & & 0,93 & 6,03 & 8,45 & 8,53 & 0,82 & 4,03 \\
\hline
\end{tabular}




\begin{tabular}{|c|c|c|c|c|c|c|c|c|c|}
\hline & & $\mathbf{H}$ & $\mathbf{M}$ & Ch-M & $\mathbf{V}$ & Ch-V & A & $\mathbf{V P}$ & SD \\
\hline \multirow[t]{2}{*}{ Macroherbívoros } & $\mathrm{N}$ & 2 & 2 & 2 & 2 & 2 & 2 & 2 & 2 \\
\hline & $\%$ & 2,26 & 2,06 & 0,46 & 1,18 & 3,06 & 5,78 & 3,67 & 3,15 \\
\hline Gasterópodos & $\mathrm{N}$ & 1 & ----- & 1 & ----- & ----- & ----- & ----- & ----- \\
\hline depredadores & $\%$ & 0,17 & & 0,06 & & & & & \\
\hline Asteroideos & $\mathrm{N}$ & 3 & ----- & 1 & ----- & 1 & ----- & 1 & 2 \\
\hline depredadores & $\%$ & 0,61 & & 0,29 & & 0,15 & & 0,41 & 0,46 \\
\hline Poliquetos & $\mathrm{N}$ & 2 & 2 & 1 & 2 & 2 & 2 & 2 & 2 \\
\hline depredadores & $\%$ & 3,01 & 2,74 & 1,50 & 10,30 & 4,81 & 7,84 & 6,94 & 9,62 \\
\hline \multirow[t]{2}{*}{ Nemertinos } & $\mathrm{N}$ & 1 & ----- & 1 & 1 & 1 & 1 & 1 & 1 \\
\hline & $\%$ & 2,00 & & 5,21 & 0,25 & 0,58 & 7,16 & 1,63 & 0,35 \\
\hline \multirow[t]{2}{*}{ Peces depredadores } & $\mathrm{N}$ & 1 & ----- & ----- & 1 & 1 & ----- & ----- & ----- \\
\hline & $\%$ & 0,04 & & & 0,25 & 0,29 & & & \\
\hline \multirow[t]{2}{*}{ Carroñeros } & $\mathrm{N}$ & 2 & ----- & ----- & 1 & ----- & ----- & ----- & ----- \\
\hline & $\%$ & 0,08 & & & 0,18 & & & & \\
\hline \multirow[t]{2}{*}{ Depositívoros } & $\mathrm{N}$ & 4 & 1 & 2 & 3 & 2 & 4 & 4 & 3 \\
\hline & $\%$ & 1,41 & 0,34 & 7,05 & 11,31 & 17,89 & 2,05 & 2,86 & 2,37 \\
\hline \multirow[t]{2}{*}{ Omnívoros } & $\mathrm{N}$ & 7 & 2 & 1 & 3 & 2 & 3 & 1 & 4 \\
\hline & $\%$ & 1,31 & 3,84 & 0,10 & 3,01 & 0,30 & 3,06 & 0,40 & 1,26 \\
\hline
\end{tabular}

\section{Relaciones entre poblamientos}

Los dos primeros ejes del DCA (Tabla 3) explicaron un $22 \%$ de la inercia de los datos; este valor no es demasiado alto pero el diagrama de ordenación correspondiente (Fig. 1) permite visualizar claramente las relaciones entre los bancos y poblamientos. Por otra parte, el largo de gradiente para el eje I (4,60 s) confirmó que el DCA era el método de análisis apropiado para el tratamiento de estos datos (ter Braak 1995). En el diagrama de ordenación para los dos primeros ejes se indican con diferentes símbolos las u.m. provenientes de los diferentes tipos de facies y poblamientos, las que se disponen de acuerdo al siguiente detalle:

a. Las u.m. de bancos puros de mejillón (círculos gris claro): hacia el extremo izquierdo del diagrama de ordenación.

b. Las u.m. de bancos de cholga puros (círculos negros): sobre el eje II desde la zona media hasta inferior del diagrama. Las u.m. de los bancos más profundos de cholga se ubican en la parte inferior del diagrama. c. Las u.m. de bancos mixtos de mejillón-cholga (círculos gris medio): ubicadas entre los dos grupos anteriores.

d. Las u.m. de bancos de vieira tehuelche (cuadrados gris claro): en la zona media superior del diagrama.

e. Las u.m. de bancos mixtos de vieira-cholga (cuadrados gris medio): entre las u.m. de bancos de cholga y vieira.

f. Las u.m. de bancos de almeja (rombos gris medio) y a las u.m. de poblamientos indiferenciados (rombos blancos) se encuentran dispersas entre las u.m. de otros grupos,

g. Las u.m. de bancos de vieira patagónica (rombos negros): en el extremo derecho del diagrama de ordenación.

No se observaron unidades muestrales que puedan indicar algún tipo de nexo entre las u.m. de bancos de vieira tehuelche y las de bancos de mejillón o los mixtos de mejillón-cholga ni entre estos últimos y los bancos mixtos de vieira-cholga. 
Tabla 3

Sumario de la ordenación DCA. Inercia total= 4,205

Summary of DCA ordination. Total inertia $=4.205$

\begin{tabular}{lcccc}
\hline \multicolumn{1}{c}{ Ejes } & $\mathbf{1}$ & $\mathbf{2}$ & $\mathbf{3}$ & $\mathbf{4}$ \\
Valores propios & 0,554 & 0,376 & 0,211 & 0,147 \\
Largos de gradiente & 4,606 & 2,789 & 3,095 & 3,334 \\
$\begin{array}{l}\text { Porcentaje acumulado de la varianza } \\
\text { de los datos de especies }\end{array}$ & 13,2 & 22,1 & 27,1 & 30,6 \\
\hline
\end{tabular}

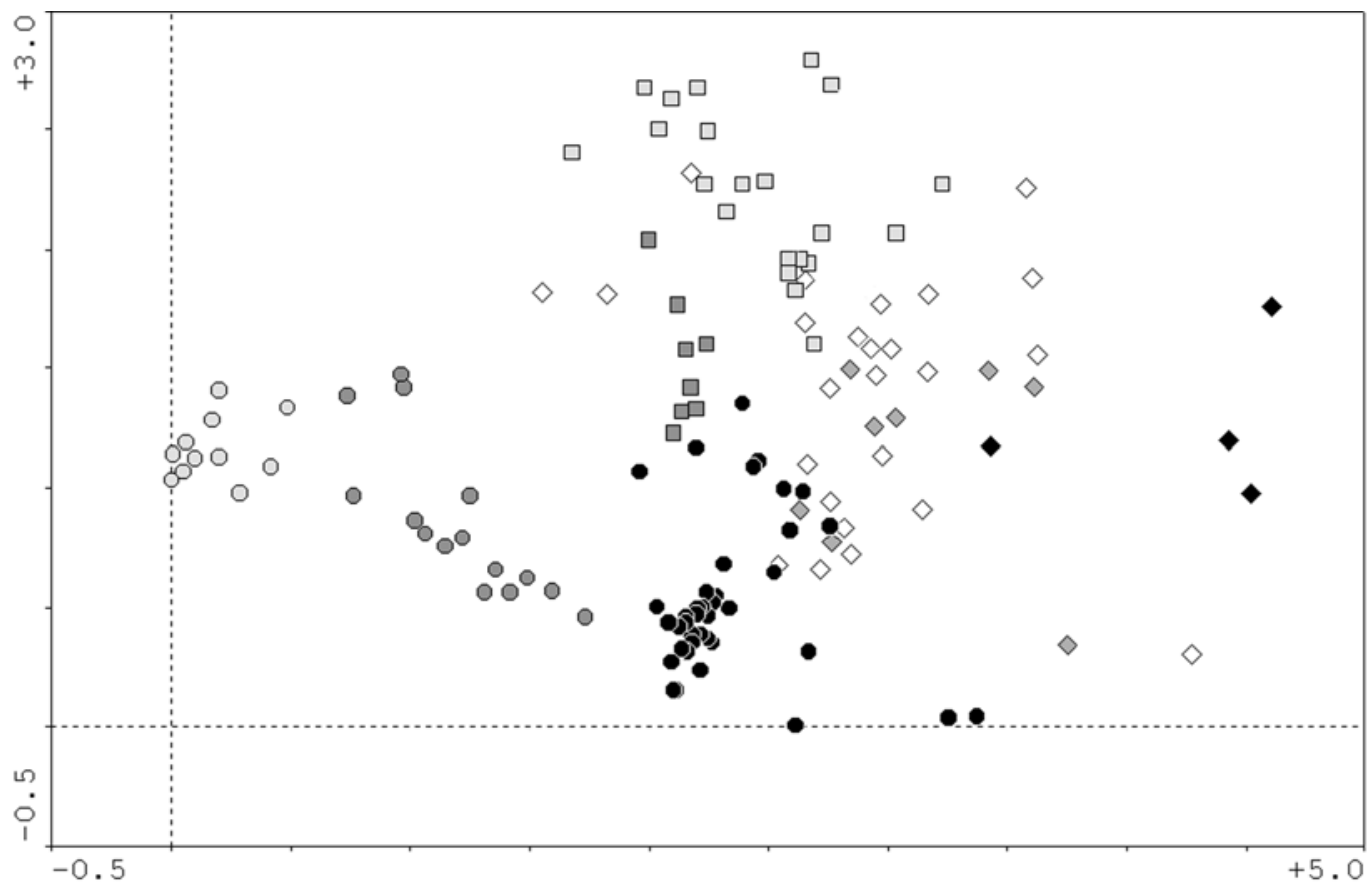

Figura 1

Análisis de correspondencias sin tendencias. Diagrama de ordenación de las unidades muestrales. Bancos de Aulacomya atra atra: círculos negros; bancos de Mytilus edulis platensis: círculos gris claro; bancos mixtos de Aulacomya-Mytilus: círculos gris medio; bancos de Aequipecten tehuelchus: cuadrados gris claro; bancos mixtos de Aulacomya-Aequipecten: cuadrados gris medio; bancos de Ameghinomya antiqua: rombos gris medio; bancos de Zygochlamys patagonica: rombos negros; poblamientos sin dominancias: rombos blancos

Detrended correspondence analysis. Ordination diagram of the sampling units. Aulacomya atra atra beds: black circles; Mytilus edulis platensis beds: light grey circles; Aulacomya-Mytilus mixed beds: medium grey circles; Aequipecten tehuelchus beds: light grey squares; Aulacomya-Aequipecten mixed beds: medium grey squares; Ameghinomya antiqua beds: medium grey diamonds;

Zygochlamys patagonica beds: black diamonds; assemblages without dominances: white diamonds 


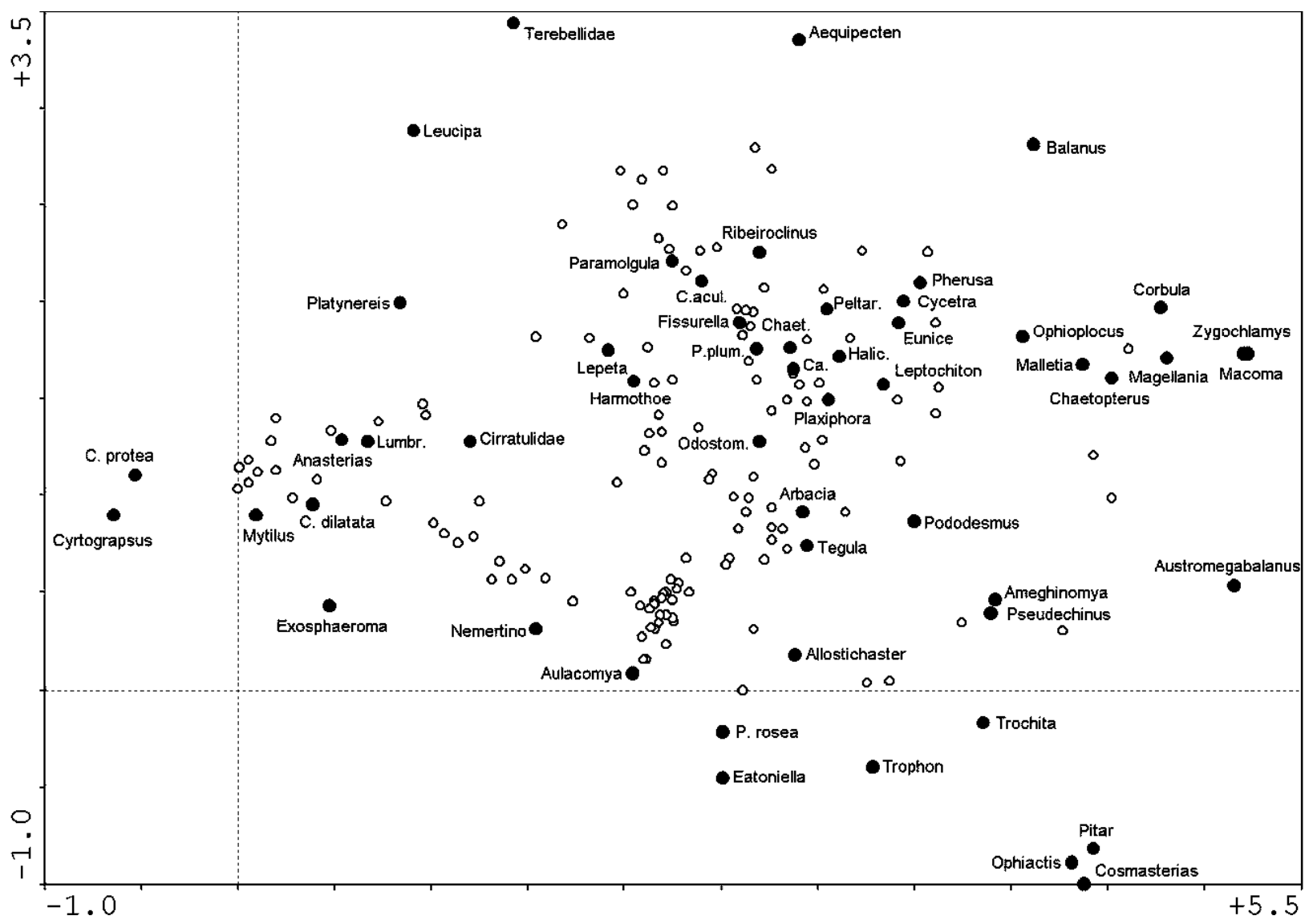

Figura 2

Análisis de correspondencias sin tendencias. Diagrama conjunto de especies (gris oscuro) y u.m. (gris claro)

Detrended correspondence analysis. Species (dark grey) and sampling units (ligth grey) joint plot

En el diagrama conjunto (“joint plot”) de ordenación de especies y u.m (Fig. 2) queda expresada en forma resumida la composición cuantitativa (porcentual, por haberse utilizado un DCA) de los grupos de u.m. en función de los puntos de especies que las rodean.

\section{Relaciones con factores ambientales}

Los resultados del análisis factorial de correspondencias entre poblamientos y factores ambientales son indicados en la Tabla 4; el porcentaje de la inercia explicada por los dos primeros ejes es del 73\%. De este análisis se obtuvo que:

a. Los bancos de cholga se hallan relacionados principalmente con sustratos de toba, profundidades entre 0 y $5 \mathrm{~m}$ y entre 15 a $30 \mathrm{~m}$ y exposiciones bajas al oleaje producido por vientos locales.

b. Los bancos de mejillón se asocian a profundidades de hasta $5 \mathrm{~m}$, sustratos con predominio de gránulos y arenas muy gruesas y exposiciones bajas al oleaje.

c. Los bancos mixtos de cholga y mejillón se asocian a profundidades de hasta $15 \mathrm{~m}$, sustratos con predominio de gránulos y arenas muy gruesas y exposiciones bajas al oleaje.

d. Los bancos de vieira tehuelche se relacionan principalmente con sustratos con predominio de gránulos y arenas muy gruesas, con profundidades entre 5 y $15 \mathrm{~m}$, exposiciones medias o altas al oleaje; secundariamente se asocian a profundidades mayores a los 30 m y sustratos de granulometría muy fina. 
Tabla 4

Análisis de correspondencias entre poblamientos y factores ambientales. Probabilidades condicionales de la tabla de datos originales. Las probabilidades condicionales más elevadas que la probabilidad incondicional (entre paréntesis a la izquierda de cada fila), se hallan marcadas en negrita e indican la correspondencia entre descripciones. Datos ranqueados sobre el primer eje. $\mathrm{CH}$, bancos de cholga, $\mathrm{M}$, bancos de mejillón; $\mathrm{CH}-\mathrm{M}$, bancos de cholga y mejillón; V, bancos de vieira tehuelche; CH-V, bancos de cholga y vieira tehuelche; A, bancos de almeja; VP, bancos de vieira patagónica; SD, poblamientos sin dominancias;

Pro1, profundidades entre 0 y 4,9 m; Pro2, profundidades entre 5 y 14,9 m; Pro3, profundidades entre 15 y 29,9 m; Pro4, profundidades de $30 \mathrm{~m}$ o más; Toba, sustrato duro; S1-S2, sustratos con predominio de las fracciones de gránulos y arenas muy gruesas; S3, sustratos con predominio de arenas finas; S4-S5, sustratos con dominancia de las fracciones de arenas muy

finas, limos y arcillas; Exp1, exposición baja al oleaje producido por vientos locales; Exp2, exposición media al oleaje producido por vientos locales; Exp3, exposición alta al oleaje producido por vientos locales

Assemblages and environmental factors correspondence analysis. Data conditional probabilities. The conditional probabilities that exceed the corresponding unconditional probabilities (between brackets at the left in each row), are in bold-face type. Data ranked over the first axis. $\mathrm{CH}$, ribbed mussel beds, $\mathrm{M}$, blue mussel beds; $\mathrm{CH}-\mathrm{M}$, ribbed mussel - blue mussel beds; V, tehuelche scallop beds; $\mathrm{CH}$ V, ribbed mussel - tehuelche scallop beds; A, clam beds; VP, patagonic scallop beds; SD, no dominance assemblages; Pro1, depths between 0 and 4,9 m; Pro2, depth between 5 and 14,9 m; Pro3, depth between 15 and 29,9 m; Pro4, depths greater than 30 m; Toba

(tuft), hard sustrata; S1-S2, granule and very coarse sand substrata; S3, fine sand substrata; S4-S5, very fine sand and silt-clay substrata; Exp1, low exposure to waves generated by local winds; Exp2, medium exposure to waves generated by local winds; Exp3,

high exposure to waves generated by local winds

\begin{tabular}{cccccccccccc}
\hline & Toba & Pro1 & Exp1 & Pro3 & Pro2 & S3 & S1S2 & S4S5 & Exp3 & Exp2 & Pro4 \\
M $(0,068)$ & 0,000 & $\mathbf{0 , 3 1 3}$ & $\mathbf{0 , 1 1 1}$ & 0,000 & 0,011 & 0,000 & $\mathbf{0 , 0 9 0}$ & 0,063 & 0,000 & 0,000 & 0,000 \\
CH $(0,292)$ & $\mathbf{1 , 0 0 0}$ & $\mathbf{0 , 4 6 9}$ & $\mathbf{0 , 4 0 4}$ & $\mathbf{0 , 6 2 5}$ & 0,281 & 0,286 & 0,153 & 0,063 & 0,143 & 0,050 & 0,063 \\
CH-M $(0,093)$ & 0,000 & $\mathbf{0 , 1 5 6}$ & $\mathbf{0 , 1 5 2}$ & 0,000 & $\mathbf{0 , 1 1 2}$ & 0,000 & $\mathbf{0 , 1 3 5}$ & 0,000 & 0,000 & 0,000 & 0,000 \\
CH-V $(0,056)$ & 0,000 & 0,000 & $\mathbf{0 , 0 9 1}$ & 0,000 & $\mathbf{0 , 0 9 0}$ & 0,000 & $\mathbf{0 , 0 7 2}$ & $\mathbf{0 , 0 6 3}$ & 0,000 & 0,000 & 0,031 \\
SD $(0,149)$ & 0,000 & 0,000 & $\mathbf{0 , 2 0 2}$ & 0,000 & $\mathbf{0 , 2 2 5}$ & $\mathbf{0 , 5 7 1}$ & $\mathbf{0 , 1 6 2}$ & 0,125 & 0,071 & 0,050 & 0,125 \\
A (0,075) & 0,000 & 0,063 & 0,030 & $\mathbf{0 , 2 5 0}$ & 0,045 & 0,000 & $\mathbf{0 , 0 8 1}$ & $\mathbf{0 , 1 8 8}$ & $\mathbf{0 , 1 1 9}$ & $\mathbf{0 , 2 0 0}$ & $\mathbf{0 , 1 2 5}$ \\
V $(0,180)$ & 0,000 & 0,000 & 0,000 & 0,125 & $\mathbf{0 , 2 3 6}$ & 0,000 & $\mathbf{0 , 2 3 4}$ & $\mathbf{0 , 1 8 8}$ & $\mathbf{0 , 4 5 2}$ & $\mathbf{0 , 5 0 0}$ & $\mathbf{0 , 2 1 9}$ \\
VP $(0,087)$ & 0,000 & 0,000 & 0,010 & 0,000 & 0,000 & $\mathbf{0 , 1 4 3}$ & 0,072 & $\mathbf{0 , 3 1 3}$ & $\mathbf{0 , 2 1 4}$ & $\mathbf{0 , 2 0 0}$ & $\mathbf{0 , 4 3 8}$ \\
\hline
\end{tabular}

e. Los bancos mixtos de cholga-vieira tehuelche se asocian a profundidades entre 5 y $15 \mathrm{~m}$, sustratos con predominio de gránulos-arenas muy gruesas o con predominio de granulometrías finas y exposiciones bajas al oleaje.

f. Los bancos de almeja se relacionan principalmente con profundidades mayores de $15 \mathrm{~m}$, sustratos con predominio de granulometrías finas y exposiciones medias o altas al oleaje.

g. Los bancos de vieira patagónica se relacionan con profundidades mayores de $30 \mathrm{~m}$, sustratos con predominio de fracciones finas o muy finas $y$ exposiciones al oleaje medias o altas.

h. Los poblamientos sin dominancias se relacionan principalmente con profundidades entre 5 y $15 \mathrm{~m}$, sustratos con granulometrías con predominio de arenas finas o más gruesas y exposiciones bajas al oleaje.

\section{Diversidad}

Un ANOVA de los valores de riqueza específica $\left(\mathrm{N}_{0}\right)$ mostró que existen diferencias altamente significativas entre facies $\left(p=3,12910^{-11}\right)$. La subsiguiente prueba de comparación múltiple entre medias encontró $(\alpha=0,01)$ que los bancos de cholga, vieira-cholga y mejillóncholga son los que presentan mayor número de especies y no presentan diferencias significativas entre ellos (Tabla 5); las facies de mejillón y poblamientos sin dominancias son los que presentaron el menor número de especies y no difirieron entre sí. Los bancos de vieira presentaron valores intermedios y no presentaron diferencias con los grupos anteriores.

Un ANOVA llevado a cabo con los valores del índice del número efectivo de especies abundantes $\left(\mathrm{N}_{1}\right)$, encontró diferencias altamente significativas entre facies $\left(p=6,24310^{-8}\right)$. Una prueba de comparación múltiple entre medias encontró $(\alpha=0,01)$ que los bancos de vieira y vieira-cholga presentaron los mayores valores, esto es el mayor número efectivo de especies abundantes y no presentaron diferencias significativas entre sí (Tabla 5); los bancos de mejillón y cholga son los que presentaron el menor número efectivo de especies abundantes y no difirieron entre sí; las facies 
de mejillón-cholga y poblamientos sin dominancias presentaron valores intermedios $y$ no difirieron significativamente del grupo de facies con $\mathrm{N}_{1}$ bajos ni con los bancos de vieira.

Un ANOVA practicado con los valores del índice del número efectivo de especies muy abundantes $\left(\mathrm{N}_{2}\right)$, encontró asimismo diferencias altamente significativas entre facies $\left(p=3,12910^{-11}\right)$. Una prueba “a posteriori” de comparación entre medias señaló la existencia de dos grupos (Tabla 5), uno con valores bajos del número efectivo de especies muy abundantes (bancos de mejillón y de cholga) y otro con valores significativamente $(\alpha=0,01)$ mayores (poblamientos sin dominancias, de vieira y de vieira-cholga). Entre ambos grupos se hallaron los poblamientos de mejillón-cholga, con valores intermedios en el número efectivo de especies muy abundantes.

Finalmente un ANOVA llevado a cabo con los valores del índice de uniformidad $\left(\mathrm{N}_{2} / \mathrm{N}_{1}\right)$ encontró diferencias altamente significativas entre facies $\left(p=1,70910^{-9}\right)$. Una prueba GT2 de comparaciones múltiples entre medias encontró diferencias altamente significativas $(\alpha=0,01)$ entre la media $(0,49)$ de los bancos de cholga y las medias $(0,69$ a 0,73$)$ de los bancos de mejillón, mejillón-cholga, vieira y poblamientos sin dominancias (Tabla 5); al respecto se recuerda que valores del índice tendientes a uno indican una baja uniformidad. Los bancos de vieira-cholga son intermedios y no difirieron significativamente de ambos conjuntos.

\section{Complejidad estructural}

La complejidad estructural de los bancos y poblamientos estudiados puede ser definida por el tipo de fijación de los individuos al sustrato, el número de capas observables, la presencia de epibiontes, etc.

a. En los bancos de cholga los individuos más cercanos al sustrato se fijan directamente a la roca y sus valvas sirven para la fijación de otras cholgas, macroalgas, diversos tipos de epibiontes y especies de la macrofauna. Especies de la meiofauna viven en los intersticios o entre los filamentos bisales. No se observa tendencia de los individuos a disponerse en varias capas, pero si a su agregación. La cholga cubre total (en el infralitoral superior) o parcialmente (en el infralitoral inferior) al sustrato; en este último caso, los espacios entre cholgas están usualmente ocupados por otras especies y raramente están desnudos. Los bancos de cholga no son exclusivos de fondos duros y pueden estar asociados a otros tipos de sustratos, particularmente aquellos donde predominan las fracciones de gravas y gránulos; en este caso los individuos más cercanos al sustrato se fijan a gránulos, trozos de conchas y gravas.

b. En los bancos de mejillones la fijación bisal se lleva a cabo principalmente a gránulos, grava, conchas o fragmentos de éstas, que se hallan presentes en su superficie o a escasa profundidad; las valvas de los animales fijados directamente al sustrato sirven a su vez de sustrato de fijación y soporte para otros mejillones, para las especies de epibiontes que viven sobre ellos y para las especies que viven en los numerosos intersticios de la matriz del banco. Existe una marcada tendencia a que los mejillones se dispongan en varias capas. La unión del conjunto de ejemplares al sustrato es relativamente débil, ya que depende estrictamente del efecto de anclaje que puedan proporcionar los gránulos utilizados en la fijación de los individuos más cercanos al sustrato.

Tabla 5

Valores promedio de los índices de diversidad de Hill en algunos de los poblamientos estudiados. N, número de u.m.; $\mathrm{s}^{2}$, varianza. Para $\mathrm{N}_{0}$ los valores han sido retransformados a partir de la transformación raíz cuadrada

Average values of Hill diversity indices in some of the studied assemblages. N, number of sampling units; $\mathrm{s}^{2}$, variance. The values of $\mathrm{N}_{0}$ have been back-transformed from square root transformation

\begin{tabular}{lcccc}
\hline \multicolumn{1}{c}{ Poblamientos (N) } & $\mathbf{N}_{\mathbf{0}}\left(\mathbf{s}^{2}\right)$ & $\mathbf{N}_{\mathbf{1}}\left(\mathbf{s}^{2}\right)$ & $\mathbf{N}_{\mathbf{2}}\left(\mathbf{s}^{\mathbf{2}}\right)$ & $\mathbf{N}_{\mathbf{2}} / \mathbf{N}_{\mathbf{1}}\left(\mathbf{s}^{2}\right)$ \\
Mejillón (11) & $5,80(0,0133)$ & $3,59(0,9438)$ & $2,81(0,5292)$ & $0,69(0,0074)$ \\
Cholga-Mejillón (15) & $10,91(0,0086)$ & $5,48(1,8769)$ & $3,92(1,5316)$ & $0,63(0,0112)$ \\
Cholga (39) & $14,06(0,2108)$ & $4,80(5,6096)$ & $3,07(3,4683)$ & $0,49(0,0231)$ \\
Cholga-Vieira (8) & $15,23(0,0282)$ & $8,39(2,2024)$ & $5,49(1,6282)$ & $0,60(0,0133)$ \\
Vieira (22) & $10,75(0,0431)$ & $7,15(2,5989)$ & $5,34((2,5067)$ & $0,73(0,0114)$ \\
Sin dominancias (24) & $8,997(0,0836)$ & $5,61(4,3067)$ & $4,55(2,9890)$ & $0,69(0,0264)$ \\
\hline
\end{tabular}


c. Los bancos mixtos de cholgas y mejillones también se presentan sobre sustratos muebles y los individuos se fijan al sustrato a través de gránulos, grava, conchas o fragmentos de éstas presentes en éste. La estructura es generalmente de varias capas y con respecto a los bancos de mejillón, hay un mayor número de epibiontes y organismos que viven en los intersticios de la matriz.

d. Los bancos de vieira tehuelche se presentan principalmente sobre fondos blandos, con preferencia en aquellos que contienen gránulos que sirven para la fijación bisal de los individuos. Las vieiras se hallan dispersas sobre el fondo y nunca forman agregaciones de varias capas, excepto en el caso de "arribazones" donde pueden acumularse en depresiones del fondo. La fijación bisal de las vieiras al sustrato es lábil y pequeños estímulos externos, producen el desprendimiento de los individuos.

e. Los bancos mixtos de cholga y vieira tehuelche se asocian a fondos blandos donde los individuos se fijan a los gránulos y restos de conchas presentes en el sustrato o a las conchas de las vieiras y cholgas. Existe una mayor tendencia a la agregación de los individuos debido a la presencia de cholgas, pero éstos nunca se disponen en capas.

f. Los bancos de almeja menos profundos se ubican sobre sustratos blandos y se asemejan en cuanto a su composición del estrato superficial a bancos de cholga de muy baja densidad. Los individuos de Ameghinomya antiqua se hallan, como es propio de esta especie, enterrados a escasa profundidad.

g. Los bancos de vieira patagónica no son accesibles a la observación directa en razón de su profundidad, sin embargo su estructura puede ser extrapolada a partir de la observación de muestras de draga poco disturbadas. Asociados a fondos de granulometría media o fina, los individuos se fijan débilmente con sus biso a gránulos, restos de valvas o a la concha de vieiras vivas. La situación observada en los mismos a partir de rastreos cualitativos es compleja, ya que además de los bancos puros de vieira patagónica, fueron encontrados bancos mixtos de esta especie con cholga, vieira tehuelche y almeja rayada. Se han observado también situaciones de transición hacia biocenosis caracterizadas por la dominancia de consumidores de depósitos.

\section{Discusión y conclusiones}

La biocenosis de raspadores-suspensívoros del golfo San José es una de las más importantes en cuanto a área ocupada (Zaixso et al. 1998). En Zaixso et al. (1998) y Zaixso (1999) se señala que esa biocenosis puede presentar diferentes aspectos de acuerdo a la dominancia relativa de sus especies y se postula que los mismos corresponderían a facies. Estas fisonomías denominadas comúnmente "bancos” de cholga, de vieira o mejillones incluyen a las facies de bancos profundos de cholga.

El parentesco biocenológico entre poblamientos resulta claro al comparar las especies constituyentes de cada uno (Tabla 1).

En los puntos siguientes se discutirán las relaciones entre la diversidad observada en los poblamientos estudiados y los siguientes factores que pueden influenciar su composición: (1) sustrato y disturbios; (2) depredación y (3) sucesión y heterogeneidad espacial.

En los ambientes caracterizados por una alta dinámica del agua (costeros o con corrientes fuertes), en los que se desarrolla la mayor parte de la biocenosis de raspadores-suspensívoros del golfo San José se encuentran dos tipos principales de sustratos: los fondos rocosos de toba y los fondos de sedimentos muebles de granulometría gruesa o media (Zaixso et al. 1998). De los resultados del presente trabajo surge que los primeros sirven, por lo general, de sustrato a los bancos de cholga, y los segundos a los bancos de mejillón, almeja y vieira tehuelche. Los bancos de vieira patagónica se encuentran a profundidades mayores que los anteriores y a diferencia de éstos se hallan asentados sobre sustratos de granulometría fina a muy fina. Estos resultados coinciden en general con los de Ciocco (1988), Bala (1989) y Zaixso (1993, 1996a, 1999).

Ante la presencia de disturbios como tormentas $\mathrm{u}$ oleaje fuerte, los bancos más susceptibles de resultar alterados serían los ubicados en aguas someras, en particular si la unión de los animales con el sustrato es de tipo débil o lábil, como ocurre en los bancos de mejillón o vieira tehuelche. Esta suposición se ve confirmada por la ocurrencia más o menos frecuente en el golfo San José de arribazones intermareales de estas especies y de su fauna y flora acompañante, las que han sido reportadas para diferentes fechas por (Ciocco (1985), Orensanz (1986) y Bala (1989).

En el caso de la vieira tehuelche se ha sugerido que la mortalidad por arribazones, considerada como la fuente más importante de mortalidad, se incrementa con la edad de los individuos, debido tanto a una disminución en la capacidad de fijación bisal con la talla como al aumento de las fuerzas de arrastre asociadas a una mayor carga de epibiontes (Orensanz et al. 1991). Luego de ocurridas las arribazones se ha podido verificar sobre los fondos disturbados, el reclutamiento de juveniles de vieira (Orensanz 1986). 
En el caso de los bancos de mejillones, las arribazones son menos frecuentes, ya que estos se hallan preferentemente en costas protegidas del oleaje producido por los vientos dominantes. Sin embargo, los bancos de la costa oeste suelen desarraigarse por la acción de mares de fondo, que entran al golfo por su boca ubicada en el ángulo NW.

Disturbios equivalentes a los citados han sido descriptos para bancos de mitílidos por Kuenen (1942), Theisen (1968), Dayton (1971) y Seed (1976).

Los bancos de cholga sobre sustratos duros no se ven mayormente afectados por este tipo de disturbios por hallarse en sitios protegidos y por poseer una matriz física fijada firmemente a la roca. En costas patagónicas más expuestas, como las del golfo San Jorge (4552'S; $\left.67^{\circ} 28^{\prime} \mathrm{W}\right)$, con temporales de mayor magnitud de los que pueden tener lugar en el golfo San José, se han observado arribazones de cholgas provenientes de bancos asentados sobre fondos de roca blanda, luego de tormentas extremadamente fuertes (Zaixso 2003) ${ }^{2}$.

También son poco afectados por tormentas o mares de fondo los bancos de almejas y vieras patagónicas del golfo San José, ya que estas especies tienen su óptimo en aguas relativamente profundas.

La interdependencia entre diversidad y estabilidad de la comunidad se puede interpretar en términos del comportamiento de la comunidad cuando sus componentes son perturbados; así una comunidad puede ser definida como estable o inestable en la medida que una perturbación del sistema tienda a amplificarse o a desaparecer (Pielou 1975). Es posible que tanto la estabilidad de una comunidad como su diversidad dependan ambas de la estabilidad ambiental, determinando la existencia de una correlación positiva entre ellas sin que una sea necesariamente la causa de la otra (Pielou 1975).

Los ambientes en los que se encuentra la biocenosis de raspadores-suspensívoros difieren entre sí fundamentalmente en el sustrato, profundidad y grado de exposición a las tormentas y mares de fondo. La interacción entre estos factores parece ser la responsable en última instancia, a través de la naturaleza de la fijación bisal al sustrato de las especies dominantes, del

\footnotetext{
2 Zaixso HE. 2003. Ecología de la cholga, Aulacomya atra atra. XXIII Congreso de Ciencias del Mar. Punta Arenas, Chile, p.51, resumen.
}

grado de resistencia a los disturbios de algunos de los poblamientos de la biocenosis.

Los valores de diversidad hallados concuerdan con la hipótesis que relaciona estabilidad y diversidad, puesto que los bancos de cholga fueron los más diversos tanto en riqueza específica como en uniformidad; los de vieira y mejillón, al igual que las u.m. de áreas indiferenciadas, fueron los menos diversos, mientras que los bancos mixtos contaron con valores intermedios de diversidad.

En los poblamientos estudiados, con excepción de los bancos de cholga, es baja o nula la presencia de gasterópodos y asteroideos depredadores (Tabla 2).

En los bancos de cholga, tanto el murícido Trophon geversianus como los asteroideos Cosmasterias lurida y Allostichaster capensis depredan preferentemente sobre Aulacomya atra atra (Zaixso datos no publicados).

La baja incidencia de depredadores en facies de la biocenosis de raspadores-suspensívoros está documentada para bancos de vieira tehuelche (Orensanz 1986, Ciocco 1988) y de mejillón (Bala 1989). Orensanz (1986) y Orensanz et al. (1991) indican al asteroideo Cosmasterias lurida, al volútido Odontocymbiola magellanica y al pulpo Enteroctopus megalocyathus como depredadores de la vieira tehuelche, si bien señalan que sus densidades son bajas y que constituyen una fuente importante de mortalidad. Estas especies no fueron halladas en las u.m. de bancos de vieiras aquí analizadas.

Se ha señalado que la depredación selectiva puede aumentar la diversidad en una comunidad, particularmente si la presa preferida es competitivamente dominante; este efecto sería mayor cuando los depredadores actúan en forma densodependiente y la intensidad de la depredación es de orden intermedio (Begon et al. 1996). En poblamientos dominados por Mytilus californianus por ejemplo, la predación de esta especie por la estrella Pisaster ochraceus crearía espacios libres que podrían ser ocupados por especies competitivamente subordinadas (Paine 1966).

De acuerdo a Connell (1975), el papel de los depredadores en la estructuración de las biocenosis sería menos importante en los sitios donde las condiciones físicas son más severas, variables o impredecibles. $\mathrm{Al}$ respecto, Orensanz (1986) ha sugerido que los procesos de arribazón que ocurren en los bancos de vieira del golfo San José, podrían ser los responsables de la escasa 
cantidad de depredadores presentes y de la baja incidencia de la depredación en los bancos.

Los organismos raspadores (por ej. Tegula patagonica, Fissurella radiosa, Chaetopleura isabellei, Lepeta coppingeri) y macroherbívoros (Arbacia dufresnei y Pseudechinus magellanicus), que son típicos de la biocenosis bajo estudio, pueden jugar un papel semejante al de los depredadores sobre la estructura de la misma al ingerir juveniles de algunas especies animales junto a su alimento habitual. Un ejemplo de este efecto se señala para los erizos $A$. dufresnei y $P$. magellanicus, en bancos circalitorales de mejillón al norte de la costa atlántica de Argentina (Penchaszadeh 1979). Ambas especies se hallan presentes en todos los poblamientos de la biocenosis de raspadoressuspensívoros del golfo.

El comportamiento de limpieza de las valvas con el pie, que fuera descripto por Theisen (1972) para Mytilus edulis y por Bruzzone (1982) para Mytilus edulis platensis (citado como Mytilus edulis chilensis), podría dar cuenta de parte de las diferencias en diversidad entre bancos de mitílidos. De acuerdo a Theisen (1972) las valvas de los individuos vivos están protegidas contra los epibiontes debido a esta actividad de limpieza, en tanto que la concha de los individuos muertos es rápidamente incrustada con algas y epifauna y los mejillones vivos circundantes se fijan activamente a ella. La cholga en cambio, cuya concha se halla generalmente cubierta de epibiontes, carecería de este mecanismo de limpieza.

Las relaciones entre los bancos de cholga y los de fondos blandos de la almeja Ameghinomya antiqua han

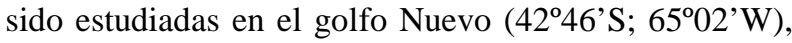
encontrándose que la acumulación de valvas de individuos muertos de venéridos posibilita el asiento de un poblamiento del mitílido Aulacomya atra atra y del alga parda Dictyota (Barrionuevo et al. 1976). Esta facies de Aulacomya-Dictyota es considerada como la etapa clímax de una sucesión (Barrionuevo et al. 1976). A su vez, el establecimiento de la cholga, con su flora y fauna acompañantes alterarían el sustrato de forma irreversible, lo que impediría el reestablecimiento de las almejas (Barrionuevo et al. 1976). Ejemplos de esta clase, donde especies propias de sustratos duros colonizan sustratos blandos, son expuestos por Connell \& Keough (1985). Observaciones llevadas a cabo en bancos de Mytilus edulis establecidos sobre sustratos blandos, indican que a medida que se incrementa la cobertura de mitílidos, los sedimentos ubicados debajo de los mejillones se hacen cada vez más anóxicos y terminan por eliminar algunas especies previamente presentes, entre ellas al bivalvo infaunal Mya arenaria
(Newcombe 1935). Si bien los aspectos anteriores no han sido estudiados en el golfo San José, es probable que existan vías de sucesión semejantes, en razón de la contigüidad espacial que presentan las facies de cholga $\mathrm{y}$ almeja y por el hecho de que comparten numerosas especies. Dado que las poblaciones de almejas en el golfo persisten a través del tiempo, es probable que al menos en aguas someras, sean las tormentas o mares de fondo las que reviertan dicha sucesión, permitiendo que los bancos de almejas puedan reinstalarse.

Una vía de sucesión entre bancos de mejillón y bancos de cholga del golfo San José ha sido sugerida por Bala (1989). Los bancos infralitorales de mejillón del golfo San José en sitios de sustratos blandos no son permanentes, apareciendo y desapareciendo con relativa frecuencia; los mismos constituyen facies donde, al menos al principio, los únicos mitílidos presentes son los mejillones. Ejemplos de estos bancos no permanentes son los observados en San Román en 1976 (Trancart 1978); en la costa oeste del golfo (El Bote-El Riacho) en 1974, isla de Los Pájaros en 1979 y punta Logaritmo en 1984 (Bala 1989). Las características de las biocenosis presentes en esos fondos antes de su colonización por parte de los mejillones no son conocidas. Dichos bancos, después de períodos variables de tiempo, de hasta varios años, terminan por desaparecer como resultado de disturbios provocados por temporales, mares de fondo o pesca excesiva. La composición específica de los bancos se puede mantener relativamente inalterada hasta el momento de la desaparición, como en el banco de fondeadero San Román (Trancart 1978) o, cuando persiste por más tiempo, como en el banco de El Bote, pueden por el contrario, mostrar modificaciones más o menos profundas (Bala 1989). La asociación entre bancos de mejillón y una baja exposición al oleaje, podría indicar que ésta es una condición importante para la persistencia de este tipo de bancos.

El hecho de que los bancos de mejillón y los mixtos de cholga-mejillón se presenten en ambientes de similares características respecto a sustrato, profundidad y exposición al oleaje, podría indicar que ambos tipos de banco son diferentes aspectos temporales de una misma facies. El mayor número de especies en los bancos mixtos respecto de los bancos de mejillón, podría deberse a un período más largo de colonización, a un aumento de los hábitat disponibles como resultado de la incorporación de conchas vacías a la matriz y/o a una alteración del sustrato por parte de los mejillones, etc.

El aumento de la riqueza específica con la edad del banco, es señalado para Mytilus edulis por Tsuchiya \& 
Nishihira (1985, 1986) y para M. californianus por Suchanek (1986). Este aumento es atribuido, en general, al incremento en la heterogeneidad espacial y en el número de microhábitat disponibles que tiene lugar a medida que los bancos envejecen (Seed \& Suchanek 1992).

A diferencia de los bancos de mejillón y mixtos de esta especie y cholga, resulta dudoso que los bancos de vieira tehuelche puedan cambiar su composición específica tendiendo a asemejarse a los poblamientos de cholga, ya que los bancos mixtos de cholga y vieira tehuelche son, al parecer, facies alternativas que se desarrollan en diferentes ambientes. Los bancos mixtos están asociados a sitios protegidos con exposiciones bajas al oleaje, mientras que los bancos de vieira se ubican en áreas expuestas. Los bancos de vieira tehuelche constituirían entonces facies de la biocenosis de raspadores y suspensívoros en áreas de disturbio permanente.

La ausencia de u.m. intermedias entre los bancos de vieira y los bancos con dominancia de mejillón que se observa como resultado del DCA, indicaría que ambas fisonomías de la biocenosis de raspadoressuspensívoros son independientes una de la otra, como también lo sugieren sus relaciones casi excluyentes con la profundidad, exposición al oleaje y tipo de sustrato.

Se desconoce si existe algún factor de disturbio importante que actúe sobre los bancos de vieira tehuelche (Aequipecten tehuelchus) y los bancos de vieira patagónica (Zygochlamys patagonica) que se hallan a profundidades mayores a los 30 metros, pudiéndose señalar que no se han registrado en el ámbito del golfo San José, arribazones de vieira patagónica.

Los aspectos discutidos pueden resumirse como sigue: (1) Los bancos de cholga sobre sustratos duros, en razón de hallarse en sitios protegidos y con una matriz física fijada firmemente a la roca, mantendrían una alta riqueza específica y una alta uniformidad. Es probable que los depredadores, raspadores $\mathrm{y}$ macroherbívoros contribuyan al mantenimiento de la diversidad observada. (2) Los poblamientos de fondos blandos de mejillones (y probablemente los de almejas rayadas), en condiciones de estabilidad evolucionarían hacia bancos mixtos con cholgas. Este cambio implicaría entre otros aspectos: (a) un aumento en la complejidad del sustrato (conchas vacías y rotas), (b) un aumento en la heterogeneidad espacial del banco y (c) un aumento en la riqueza específica. Estos bancos mixtos al quedar sujetos a disturbios físicos revertirían el proceso de sucesión dejando grandes espacios del bentos, disponibles potencialmente para el reclutamiento de las mismas especies. (3) Los bancos de vieira tehuelche serían propios de ambientes permanentemente disturbados por el oleaje producto de vientos locales y poseen características de baja uniformidad y baja riqueza específica. Con disturbios intensos e infrecuentes los bancos serían eliminados del sitio que ocupaban ocasionándose arribazones de las especies desprendidas y quedando libre el sustrato para un potencial reclutamiento de las mismas especies. (4) Los bancos mixtos de cholga-vieira tehuelche son facies alternativas a los bancos de vieira y a diferencia de éstos tienen lugar en sitios protegidos.

La composición, diversidad y estructura de los poblamientos identificados en la biocenosis de raspadores y suspensívoros del golfo San José, así como sus relaciones con los factores ambientales y de los poblamientos entre sí son coherentes con el anterior esquema hipotético. Sin embargo la confirmación de este esquema requiere del seguimiento detallado de varias de estas facies a lo largo de varios períodos anuales.

\section{Agradecimientos}

Se desea dejar constancia del agradecimiento del autor a los comentarios y correcciones de los dos revisores anónimos, los cuales contribuyeron eficazmente al mejoramiento de este manuscrito.

\section{Literatura citada}

Alatalo RV \& R Alatalo. 1977. Components of diversity: Multivariate analysis and interaction. Ecology 58: 900-906.

Bala LO. 1989. Biología y ecología del mejillón (Mytilus edulis platensis) en el golfo San José, provincia del Chubut. Tesis doctoral, Ciencias Naturales, Universidad Nacional de La Plata, 278 pp.

Barbault R. 1981. Ecologie des populations et des peuplements. Masson Ed., Paris, 200 pp.

Barrionuevo DN, M Schuldt \& M Verdinelli. 1976. Nuevas consideraciones ecológicas sobre la cholguera de Punta Loma, Golfo Nuevo, Chubut. Revista Museo de La Plata (Nueva Serie), Zoología 118: 167-181.

Begon M, JL Harper \& CR Townsend. 1996. Ecology: Individuals, populations and communities. Blackwell Science, Oxford, 1068 pp. 
Boraso de Zaixso AL, H Zaixso \& G Casas. 1999. Asociaciones de algas bentónicas submareales del golfo San José (Chubut, Argentina). Physis (Buenos Aires) Sección A 57: 17-27.

Bruzzone JH. 1982. Funciones del pie en Mytilus edulis chilensis (Hupe). Physis (Buenos Aires), Sección A 41: 5161.

Cancino J \& R Becerra. 1978. Antecedentes sobre la biología y tecnología del cultivo de Aulacomya ater (Molina, 1782), (Mollusca: Mytilidae). Biología Pesquera, Chile 10: 27-45.

Carriquiriborde L, C Borzone, Z Lizarralde, A Pombo, $\mathbf{R}$ Manrique \& M Ichazo. 1983. Aspectos biocenológicos del Golfo Nuevo (Chubut - Argentina). Informe Interno, Centro Nacional Patagónico, Puerto Madryn, 12 pp.

Charpy C \& L Charpy. 1977. Biomasse phytoplanctonique, production primaire et facteurs limitant la fertilité des eaux du golfe "San José" (Péninsule Valdès, Argentine). Thèse de Doctorat de $3^{\text {eme }}$ Cycle, Océanologie, Université d'AixMarseille II, 185 pp.

Ciocco NF. 1985. Biología y ecología de Chlamys tehuelchus d'Orbigny en el golfo San José (Provincia del Chubut, República Argentina) (Pelecypoda, Pectinidae). Tesis doctoral, Ciencias Naturales, Universidad Nacional de La Plata, 406 pp.

Ciocco NF. 1988. Observaciones sobre la ecología del molusco bivalvo Chlamys tehuelchus (d’Orb.) en el golfo San José (Chubut, Argentina). I. Análisis biocenótico. Neotrópica 34 (91): 3-22.

Ciocco NF, CA Borzone \& DE Ruzzante. 1983 Observaciones sobre el comportamiento de fijación de Chlamys tehuelchus d'Orbigny en bancos naturales. Memorias de la Asociación Latinoamericana de Acuicultura 5 (29): 271-275.

Connell JH. 1975. Some mechanisms producing structure in natural communities: a model and evidence from field experiments. En: ML Cody \& JM Diamond (eds) Ecology and evolution of communities, pp. 460-490. Belknap, Cambridge.

Connell JH \& MJ Keough. 1985. Disturbance and patch dynamics of subtidal marine animals on hard substrata. En: Pickett STA \& PS White (eds) The ecology of natural disturbance and patch dynamics, pp. 125-151. Academic Press, San Diego.

Dayton PK. 1971. Competition, disturbance and community organisation: the provision and subsequent utilisation of space in a rocky intertidal community. Ecological Monographs 41: 351-389.
Escofet A, JM Orensanz, SR Olivier \& V Scarabino. 1978. Biocenología bentónica del golfo San Matías (Rio Negro, Argentina): Metodología, experiencias y resultados del estudio ecológico de un gran espacio en América Latina. Anales Centro Ciencias del Mar y Limnología Universidad Autónoma de México 5 (1): 59-82.

Hill MO. 1973. Diversity and eveness: A unifying notation and its consequences. Ecology 54: 427-432.

Kuenen DJ. 1942. On the distribution of mussels on the intertidal flats near den Helder. Archives Néerlandaises Zoologie 6:117-160.

Legendre $\mathbf{P}$ \& L Legendre. 1998. Numerical ecology. Second edition. Elsevier, Amsterdam, 853 pp.

Ludwig JA \& JF Reynolds. 1988. Statistical ecology. A primer on methods and computing. John Wiley \& Sons, New York, 337 pp.

Magurran AE. 1988. Ecological diversity and its measurement. Princeton University Press, Princeton, 179 pp.

Newcombe CL. 1935. A study of the community relationships of the sea mussel, Mytilus edulis L. Ecology 16: 234-243.

Olivier SR, I Kreibhom de Paternoster \& R Bastida. 1966. Estudios biocenóticos en las costas de Chubut (Argentina) I. Zonación biocenológica de Puerto Pardelas (Golfo Nuevo). Boletín Instituto Biología Marina, Mar del Plata (10), 74 pp.

Orensanz JM. 1986. Size, environment and density: the regulation of a scallop stock and its management implications. Canadian Special Publications in Fisheries and Aquatic Science 92: 195-227.

Orensanz JM, M Pascual \& M Fernández. 1991. Argentina. En: SE Shumway (ed) Scallops: Biology, ecology and aquaculture, pp. 981-999. Elsevier, Amsterdam.

Otaegui AV \& HE Zaixso. 1974. Distribución vertical de los moluscos marinos del litoral rocoso de la ría de Puerto Deseado (Santa Cruz, Argentina). Una guía para reconocer los diferentes pisos y horizontes litorales. Physis, Secc. A 33 (86): 321-334.

Paine RT. 1966. Food web complexity and species diversity. American Naturalist 100: 65-75.

Penchaszadeh PE, MA Burgos \& MA Scilingo. 1974. Situación actual de los conocimientos biológicos y ecológicos de la cholga (Aulacomya ater; Mollusca Mytilidae). Perspectivas de su explotación y cultivo. II Reunión del Grupo Internacional de Coordinación de los Océanos Australes. Consejo Nacional de Investigaciones Científicas y Técnicas, Buenos Aires (1974): 49-56. 
Penchaszadeh PE. 1979. Estructura de la comunidad y procesos que la determinan en bancos circalitorales de mejillón Mytilus platensis. En: Ecología bentónica y sedimentación de la plataforma continental del Atlántico Sur: 131-147. Memorias Seminario UNESCO, Montevideo.

Pérès JM \& J Picard. 1964. Nouveau manuel de bionomie benthique de la Mer Méditerranée. Recueil des Travaux Station Marine d’Endoume 31: 1-137.

Pielou EC. 1975. Ecological diversity. Wiley-Interscience, Nueva York, 165 pp.

Seed R. 1976. Ecology. En: BL Bayne (ed) Marine mussels: Their ecology and physiology, pp. 13-65. Cambridge University Press, Cambridge.

Seed R \& TH Suchanek. 1992. Population and community ecology of Mytilus. En: E Gosling (ed) The mussel Mytilus: Ecology, physiology, genetics and culture, pp. 87169. Elsevier, Amsterdam.

Sokal RR. \& FJ Rholf. 1997. Biometry. The principles and practice of statistics in biological research. Third Edition. W. H. Freeman \& Company, New York, 887 pp.

Suchanek TH. 1986. Mussels and their role in structuring rocky shore communities. En: PG Moore \& R Seed (eds) The ecology of rocky coast, pp. 70-96. Columbia University Press, New York.

ter Braak CJF. 1995. Ordination. En: RHG Jongman, CJF Ter Braak \& OFR Van Tongeren (eds). Data analysis in community and landscape ecology, pp. 91-173. Cambridge University Press, Cambridge.

ter Braak CJF. \& P Smilauer. 1998. Canoco reference manual and user's guide to Canoco for Windows: Software for canonical community ordination (version 4). Microcomputer Power (Ithaca, New York), 352 pp.

Theisen BF. 1968. Growth and mortality of culture mussels in the Danish Wadden Sea. Meddelelser fra Danmarks Fiskeri-og Havundersogelser, N. S. 6: 47-78.

Theisen BF. 1972. Shell cleaning and deposit feeding in Mytilus edulis L. (Bivalvia). Ophelia 10: 49-55.

Trancart M. 1978. Biologie et possibilités d'exploitation de Mytilus platensis d’Orb. dans le Golfe San José, Péninsule Valdès, Argentine. Thèse de Doctorat de $3^{\text {eme }}$ Cycle. U.E.R. des Sciences de la mer et l'environnement (Université D’Aix-Marseille II), 86 pp.
Tsuchiya M \& M Nishihira. 1985. Islands of Mytilus as habitat for small intertidal animals: Effect of island size on community structure. Marine Ecology Progress Series 25: 71-81.

Tsuchiya M \& M Nishihira. 1986. Islands of Mytilus as habitat for small intertidal animals: Effect of Mytilus age structure on the species composition of the associated fauna and community organization. Marine Ecology Progress Series 31: 171-178.

Urquiola de De Carli, MJ. \& SA Aramayo. 1970. Observaciones ecológicas sobre la cholguera de Punta Loma (Golfo Nuevo). Neotrópica 16 (49): 35-41.

Zaixso HE. 1993. Distribución de Chlamys tehuelcha y Chlamys patagonica (Bivalvia, Pectinidae) en el golfo San José (Chubut, Argentina) en función de la profundidad y del sustrato. Physis (Buenos Aires), Sección A 51 (120121): 1-11.

Zaixso HE. 1996a. Distribución y abundancia de bivalvos de fondos blandos submareales del golfo San José y sur del golfo San Matías. Medio Ambiente (Chile) 13: 97-113.

Zaixso HE. 1996b. Asociaciones de moluscos bentónicos submareales del Golfo San José y sur del Golfo San Matías (Chubut, Argentina). Physis (Buenos Aires), Sección A 54 (126-127): 1-21.

Zaixso HE. 1999. Distribución submareal del mitílido Aulacomya atra atra (Molina) en el Golfo San José (Argentina) en relación a la profundidad, características del fondo y condiciones hidrográficas. Physis (Buenos Aires), Sección A 57 (132-133): 1-10.

Zaixso HE. \& CT Pastor. 1978. Observaciones sobre la ecología de los mitílidos de Puerto Deseado. I. Distribución y análisis biocenótico. Ecosur 4: 1-46.

Zaixso HE, AL Boraso \& JJ López Gappa. 1979. Observaciones sobre la distribución de los organismos en las costas rocosas de los alrededores de Ushuaia. Ecosur 5: 119-130.

Zaixso HE, ZI Lizarralde, CT Pastor, E Gómes Simes, E Romanello \& G Pagnoni. 1998. Distribución espacial del macrozoobentos submareal del Golfo San José (Chubut, Argentina). Revista de Biología Marina y Oceanografía 33 (1): 43-72. 